\title{
Políticas de investigación para las universidades: La financiación de centros de investigación en Galicia
}

\section{Research policies for universities: The funding of research centers in Galicia}

\author{
Manuel Pereira-Puga \\ Instituto de Políticas y Bienes Públicos - IPP-CSIC (España) \\ ORCID: https://orcid.org/0000-0002-1677-2318 \\ manuel.pereira@csic.es
}

\section{NOTA BIOGRÁFICA}

Trabaja en el Instituto de Políticas y Bienes Públicos (IPP) del CSIC, donde investiga en el ámbito de las políticas públicas de Investigación y Desarrollo $(I+D)$ y de educación superior universitaria, así como en el de la política social. Es Doctor en Sociología por la Universidad de A Coruña, gracias a una beca del Programa Nacional de Formación de Profesorado Universitario (FPU).

\author{
Luis Sanz-Menéndez \\ Instituto de Políticas y Bienes Públicos - IPP-CSIC (España) \\ ORCID: https://orcid.org/0000-0002-6869-8105 \\ luis.sanz@csic.es
}

\section{NOTA BIOGRÁFICA}

Profesor de investigación del CSIC en el Instituto de Políticas y Bienes Públicos (IPP). Doctor por la Universidad Complutense de Madrid (UCM), ha publicado libros y más de un centenar de trabajos sobre los sistemas y políticas de ciencia, tecnología e innovación en revistas como: Research Policy, Minerva, Research Evaluation, Science and Public Policy, Technological Forecasting and Social Change, Scientometrics, Regional Studies, Public Understanding of Science o PLOS One.

\section{RESUMEN}

La financiación de la investigación es una herramienta de los gobiernos para promover cambios en las universidades. Los gobiernos han intentado guiar «a distancia» a las universidades cambiando las formas de financiación y potenciando la evaluación. El artículo analiza un instrumento de financiación de centros universitarios de I+D de la Xunta de Galicia, su fundamentación lógica y los mecanismos a través de los cuales se promueve el cambio institucional. Asimismo, analiza también cómo los resultados perseguidos se ven condicionados por los instrumentos existentes y por las restricciones institucionales. El estudio confirma que el avance en los objetivos de un instrumento puede verse limitado port otros, en ausencia de coherencia de la cartera de instrumentos; y también que las limitaciones institucionales, derivadas del modelo de gobernanza universitaria, pueden convertirse en obstáculos para la transformación de las estructuras de investigación. Se concluye que los programas de financiación competitiva de centros de I+D pueden tener una capacidad transformadora, en ocasiones a través de cambios no esperados por los decisores públicos; pero que la influencia del modelo de gobernanza, del resto de instrumentos y el comportamiento de los actores condiciona la intensidad y la dirección de los cambios.

\section{PALABRAS CLAVE}

Financiación de la investigación; universidades; políticas regionales de I+D; instrumentos de promoción de la ciencia. 


\begin{abstract}
Research funding is a tool for governments to promote change in universities. Governments have tried to steer universities "from a distance» by changing the ways in which they are funded and enhancing evaluation. The article analyses a funding instrument for university R\&D centres of the Xunta de Galicia, its rationale and the mechanisms through which change is promoted. It also analyses how the outcomes pursued are conditioned by the existing instruments and by institutional constraints. The study confirms that progress in the objectives pursued by one instrument may be limited by others instruments, in the absence of coherence in the instrument-mix; also that institutional constraints, derived from the university governance model, may hinder the transformation of university research structures. It is concluded that competitive funding schemes for R\&D centres can have a transformative capacity, sometimes through changes not intended by public decision-makers; but the influence of the governance model, other instruments and actors are decisive in the intensity and direction of the changes.
\end{abstract}

\title{
KEYWORDS
}

Research funding; universities; regional R\&D policies; instruments for the promotion of science.

\section{SUMARIO}

INTRODUCCIÓN. 1. MARCO ANALÍTICO Y PREGUNTAS DE INVESTIGACIÓN. 1.1. LA FINANCIACIÓN GUBERNAMENTAL DE LAS UNIVERSIDADES Y LA I+D UNIVERSITARIA. 1.2. LA FINANCIACIÓN COMPETITIVA DE CENTROS UNIVERSITARIOS DE INVESTIGACIÓN. 1.3. GOBIERNOS, DECISORES Y CAMBIO UNIVERSITARIO. 1.4 ENFOQUE ANALÍTICO Y PREGUNTAS DE INVESTIGACIÓN. 2. EL CASO DE ESTUDIO. 3. APROXIMACIÓN METODOLÓGICA. 4. LA FINANCIACIÓN DE LA UNIVERSIDAD Y DE SU INVESTIGACIÓN POR LA XUNTA DE GALICIA. 4.1. LA FINANCIACIÓN REGULAR DE LAS UNIVERSIDADES GALLEGAS. 4.2. LA FINANCIACIÓN COMPETITIVA DE LA I+D UNIVERSITARIA Y SUS INSTRUMENTOS. 5. LA FINANCIACIÓN AUTONÓMICA DE LOS CENTROS UNIVERSITARIOS DE INVESTIGACIÓN. 5.1. ANTECEDENTES. 5.2. EL PROGRAMA DE CENTROS DE INVESTIGACIÓN SINGULARES DEL SUG. 5.3. LOS CENTROS DE INVESTIGACIÓN UNIVERSITARIOS SELECCIONADOS Y FINANCIADOS. 6. ANÁLISIS Y RESULTADOS. 6.1. LOS MECANISMOS DEL PROGRAMA. 6.2. LOS OBSTÁCULOS AL AVANCE DE LOS OBJETIVOS DEL PROGRAMA. 6.2.1. La influencia de la gobernanza y los arreglos institucionales. 6.2.2. Interacciones entre actores y entre instrumentos. 7. CONCLUSIONES. AGRADECIMIENTOS. ANEXO. REFERENCIAS BIBLIOGRÁFICAS.

\section{INTRODUCCIÓN}

En los últimos treinta años se han observado cambios importantes en las relaciones entre los gobiernos y las universidades a escala internacional (Olsen, 2007). Se ha utilizado la expresión "guiar a distancia» (Kickert, 1995) o «guiar desde la distancia» (Marginson, 1997) para describir la acción de los gobiernos. Por un lado, se han introducido modelos de financiación pública condicional de la mano de la Nueva Gestión Pública (Bleiklie, et al., 2011; De Boer et al., 2007) que, en muchos países, incluso se asocian a indicadores de resultados (Hicks, 2012). Por otro lado, la financiación competitiva de las actividades de I+D, tradicionalmente dirigida a investigadores individuales, se ha desplazado hacia la financiación de centros de investigación o de entidades supragrupales (Bozeman y Boardman, 2003). Dado que la «autonomía universitaria» está constitucionalizada, los instrumentos y las modalidades de financiación se han convertido en herramientas importantes para la coordinación, transformación y el cambio de los sistemas universitarios desde «fuera» (Braun, 1993; Rip, 1994). A la vez, constituyen elementos determinantes de las decisiones estratégicas y las prácticas organizativas de las universidades (Strehl et al., 2007, p. 13).

En España estas tendencias se han plasmado de forma limitada. Ello se ha debido a la tradicional separación, en las Administraciones Públicas (AA.PP), de las políticas universitarias y de Investigación y Desarrollo (I+D) y a la transferencia de las competencias universitarias a las Comunidades Autónomas, que han reproducido inicialmente este esquema competencial. Las Leyes de Reforma Universitaria (1983) y de Fomento y Coordinación General de la Investigación Científica y Técnica (1986) promovieron un marco regulatorio articulado y coherente (Sanz-Menéndez, 1997), por lo que las políticas universitarias y de I+D pueden verse como un dominio unitario de las políticas públicas, aunque en algunas AA.PP pueden estar separadas. 
Esta situación singular ha hecho, en opinión de muchos responsables gubernamentales, que las políticas de investigación se hayan convertido en el instrumento privilegiado de los gobiernos para la transformación de las universidades. Así, las políticas de I+D han considerado la situación de la universidad y sus problemas como parámetros en la definición de los instrumentos; véase, por ejemplo, el programa Ramón y Cajal de contratación de investigadores (Cruz-Castro y Sanz-Menéndez, 2005), en el ámbito de los recursos humanos, o el diseño de los sistemas de evaluación de la investigación (Cruz-Castro y Sanz-Menéndez, 2007).

En este artículo se estudian las políticas de I+D como dispositivos gubernamentales para promover el cambio, la adaptación y la mejora en las universidades. En particular, se analiza la política científica del gobierno regional de Galicia (Xunta de Galicia) y, especificamente, uno de sus instrumentos: el programa de centros de investigación (de excelencia) del Sistema Universitario de Galicia (SUG). El caso gallego nos permite, gracias a la configuración competencial vigente desde hace casi veinte años, examinar una política de $\mathrm{I}+\mathrm{D}$ destinada en exclusiva a las universidades y definida con el objetivo de transformarlas a través de la creación y consolidación de centros de I+D universitarios.

El objetivo de este artículo es mejorar la comprensión sobre cómo los gobiernos intentan transformar las universidades a través de políticas de I+D; y, específicamente, cómo estas últimas se ven afectadas por otros programas de financiación existentes, en el marco de las carteras de instrumentos (Capano et al., 2020), así como por las restricciones institucionales y las relaciones entre los actores que se derivan del modelo de gobernanza universitaria (Lepori, 2011).

El estudio ofrece un enfoque novedoso. Se identifican los mecanismos, dimensiones organizativas e incentivos sobre los que el programa quiere actuar y se describen barreras a la efectividad del mismo, en términos de los efectos de otros instrumentos de política autonómica y de las limitaciones que el modelo de gobernanza universitaria crea. La aproximación secuencial facilita el análisis de procesos difícilmente abordables desde otra perspectiva, debido a la complejidad derivada de las interacciones entre políticas públicas, modelo de gobernanza universitaria e incentivos individuales de los actores en el sistema.

El artículo se organiza del siguiente modo. La siguiente próxima se dedica al marco conceptual y teórico sobre el que se ha construido el análisis, así como a las preguntas de investigación. En la sección dos se presenta el caso y se señala su utilidad para contrastar empíricamente algunos de los argumentos planteados. La sección tres define la aproximación metodológica. La sección cuatro explica el marco de financiación del sistema universitario gallego y la cartera de instrumentos de apoyo a la I+D universitaria. La sección cinco describe el programa de centros y sus resultados. La sección seis presenta los principales resultados del estudio. Y, finalmente, la sección siete incluye algunas conclusiones y recomendaciones, así como futuras líneas de investigación.

\section{MARCO ANALÍTICO Y PREGUNTAS DE INVESTIGACIÓN}

La investigación sobre la influencia o los efectos de la financiación (y sus cambios en el tiempo) se puede asociar a los estudios de "educación superior» y de "políticas de ciencia y tecnología». Los siguientes sub-apartados enmarcan el análisis del programa de centros de investigación de Galicia en base al conocimiento disponible en estas dos áreas.

\subsection{La financiación gubernamental de las universidades y la I+D universitaria}

En materia de políticas universitaria y de I+D los gobiernos han actuado en diferentes frentes: modificación de los instrumentos regulatorios, por ejemplo, con el fin del tenure track en las universidades de Reino Unido en 1988 (OECD, 2008); integración de los institutos de investigación gubernamentales en las universidades, como en Dinamarca en 2007 (Aagaard et al., 2016); o el desarrollo de sistemas de evaluación de resultados y control de calidad (Whitley, 2007). Pero, sobre todo, han cambiado las formas de financiación de las universidades, los investigadores y los centros. Estos cambios se han producido en el marco de la transformación de la gobernanza universitaria, derivada de la Nueva Gestión Pública (De Boer et al., 2007). Ésta, en general, se ha abordado desde una perspectiva en la que dominaba el concepto de gobernanza del sistema, vinculado en origen a la ciencia política (Baldridge, 1971), tanto en los estudios de políticas de ciencia (Borrás, 2012) como en su aplicación al propio caso español (Arenilla, 2012).

Muchos países han cambiado el modelo por el que financian de forma recurrente las universidades públicas y se han creado esquemas de financiación pública basados en resultados (Performance-based funding) (Zacharewicz et al., 2019), siendo la investigación una dimensión clave de los resultados medibles. 
De este modo, la financiación de la universidad en su conjunto ha pasado a depender de la calidad y de los resultados, en un marco de refuerzo de la «autoridad» de los responsables universitarios, frente a los miembros de la comunidad académica. Aunque hay importantes diferencias entre los países, las universidades, como organizaciones, generalmente han aumentado también su nivel de autonomía frente a los gobiernos.

En España la transformación de la financiación pública universitaria ha cambiado menos. Las competencias en educación superior están transferidas a las CC.AA, por lo que tampoco es posible hablar de un único modelo de financiación (Hernández y García, 2015; Pérez-Esparrells et al., 2018). Podría decirse que hay dos tipos: CC.AA que reparten los fondos en base a los presupuestos liquidados de las universidades, de acuerdo a una senda pasada e incremental, y CC.AA que usan, o utilizaron temporalmente, algún tipo de fórmula para asignarlos en función de indicadores objetivables, generalmente como resultado de Planes Estratégicos (García-Aracil, 2013). En ambos casos, los análisis detectan un patrón evolutivo de carácter incremental (Larrán-Jorge y García-Correas, 2015).

En materia de financiación de la I+D se ha constatado, adicionalmente, que los gobiernos han aumentado la financiación competitiva de la I+D, asignada a través de proyectos y con evaluaciones ex ante (Van Steen, 2012), frente a la tradicional concesión directa a entidades públicas dependientes del gobierno. Se ha promovido la financiación, a un grupo de individuos, dentro de una organización, condicionada al desarrollo de actividades específicas en un período determinado de tiempo y tras la evaluación de una solicitud (Jonkers y Zacharewicz, 2016). En este contexto, las élites académicas implicadas en la asignación de los fondos competitivos desde las agencias públicas se convierten en un actor que aumenta su poder en el sistema (Whitley, 2011).

\subsection{La financiación competitiva de centros universitarios de investigación}

Asimismo, dentro del modelo de financiación competitiva de la I+D, tanto a nivel internacional como en España, se han desarrollado políticas específicas dirigidas a «subunidades» universitarias o de promoción de «centros de excelencia» (Cremonini et al., 2018; Hellström, 2011; Hellström, 2018; OECD, 2014). Estas intervenciones gubernamentales están orientadas a mejorar la calidad de la investigación, aumentar la productividad y el impacto socio-económico o promover la vinculación con el tejido productivo. Generalmente, se asume que estos programas de centros permiten la financiación a medio y largo plazo e, indirectamente, promueven la mejora del sistema universitario y científico nacional (OECD, 2014).

Sin embargo, más allá de los efectos positivos esperados y percibidos sobre esta modalidad de financiación (véase OECD, 2014), se sabe poco sobre los procesos y mecanismos a través de los que estas intervenciones gubernamentales esperan transformar las estructuras de I+D de las universidades. De hecho, hasta ahora el estudio de los centros de investigación se ha enfocado más a la propia caracterización de los mismos y la construcción de tipologías y taxonomías (véase Cruz-Castro et al., 2020). Algunos opinan que la investigación reciente adolece de falta de perspectiva temporal, imprescindible para describir los efectos de las intervenciones en los sistemas, así como de un marco conceptual sobre los instrumentos, lo que dificulta la comprensión de las interacciones entre instrumentos y entre actores (Cocos y Lepori, 2020) y, por tanto, el análisis de los efectos de los mismos en las estructuras universitarias.

\subsection{Gobiernos, decisores y cambio universitario}

En las universidades, las decisiones sustantivas sobre las actividades se delegan en los «profesionales». Adicionalmente, su modelo de organización se basa en la autonomía relativa de la política y está asociado al modelo colegial o participativo (Sahlin y Eriksson-Zetterquist, 2016), casi democrático o, como se ha calificado para España, «semipresidencial» (Benitez-Amado, 2019; Martínez, 2012). Debido a este complejo marco, caracterizado por la fragmentación de la autoridad en los modelos multinivel y donde conviven múltiples actores universitarios con preferencias diferentes, se ha llegado a asumir que a lo máximo que puede aspirar un gobierno es a influir o a guiar a las universidades.

Por su parte, en los análisis de los instrumentos se suele dar por sentada la racionalidad de los actores gubernamentales, asumiendo el supuesto de acciones intencionales (Howlett, 2009) y la coherencia de sus intervenciones. No obstante, desde el análisis de las carteras de instrumentos (Hood, 1983), otros han cuestionado su coherencia (May et al., 2005; May et al., 2006) o el mix de políticas (Capano, 2018; Capano et al., 2020).

En lo relativo a los supuestos básicos para el análisis de los policy-makers, en este artículo no se interpreta su comportamiento a través de una teoría basada en expectativas racionales (Simon, 1979). En esta investigación no sostenemos que la política e instrumentos hayan sido el resultado de las acciones inten- 
cionales y/o racionales de los decisores, como asume el modelo racional, aunque para la disección de los objetivos de los instrumentos sea importante esta idea.

Más bien, nuestra aproximación se alinea con la idea de que el diseño de los instrumentos se hace en términos de un "ajuste continuo» y de estrategias de "salir del paso» (Lindblom, 1959), basadas en preferencias -en ocasiones ambiguas y cambiantes-, en la información y recursos limitados y en un entorno político variable, entre otras cuestiones. También asumimos la existencia de visiones sobre qué cambios se buscan en el sistema y que, con frecuencia, imitan (Hedström, 1998) o se inspiran en modelos surgidos en actuaciones similares (Rose, 2005).

Como señala Peters (2011), el problema es el ajuste entre los objetivos (con frecuencia ambiguos e inconmensurables) y los diversos niveles del sistema complejo con los que el instrumento interacciona. Adicionalmente, sostenemos que el resultado (la política de I+D para las universidades) es un producto evolutivo, donde el ajuste mutuo determina los resultados (Cohen et al., 1972; Kingdon, 1996), y en el que el aprendizaje en la práctica (learning by doing o learning from the practice) (en línea con Bennett y Howlett, 1992) es importante.

\subsection{Enfoque analítico y preguntas de investigación}

En la investigación ha sido habitual ignorar los atributos de los instrumentos de financiación, o a quién financian (la universidad en conjunto, alguna de sus subunidades o a los investigadores individuales) y, sobre todo, qué factores facilitan u obstaculizan sus efectos esperados y cuál es la influencia de los contextos locales en los que operan estos instrumentos favoreciendo o no su eficacia. Es por ello que nos centramos en el análisis de los mecanismos sobre los que el programa espera actuar, sobre las restricciones institucionales o las barreras que las reglas del juego (gobernanza) imponen, así como sobre la posible incoherencia de abordar las actuaciones desde la perspectiva de un único instrumento, por central que sea para el avance de los objetivos.

El enfoque analítico se desarrolla en pasos. En primer lugar, se han identificado las dimensiones críticas de los centros de investigación sobre las que el programa quiere actuar (recursos humanos, financiación y sostenibilidad, estructura organizativa, liderazgo científico de la dirección, y coordinación científica para el uso de instalaciones y equipamientos). A continuación (en la tabla 1), dado que el programa se asocia al mito institucional de los "centros de excelencia»", se presentan los atributos asociados a esas dimensiones, sobre los que se espera que el programa actúe, que se utilizan como guía para el análisis empírico del caso.

Los atributos de las tres primeras dimensiones son: (1) la capacidad para atraer investigadores punteros y la existencia de procesos de reclutamiento altamente competitivos; (2) la viabilidad para operar a largo plazo y (3) la autonomía organizativa (Balderston, 1995). Adicionalmente, dado que una dimensión clave para analizar los centros de investigación es el liderazgo científico y la capacidad de dirección estratégica (CruzCastro y Sanz-Menéndez, 2018), se incluye (4) la existencia de mecanismos que fortalezcan la autoridad científica del director del centro. Finalmente, se incorpora (5) la mejora de las instalaciones y equipamientos y de su uso como condiciones esperables en un centro de excelencia (Cremonini et al., 2018).

Una vez hecho esto, han usado estas dimensiones para analizar los efectos de la gobernanza universitaria y los arreglos institucionales, así como de las interacciones entre instrumentos, y entre los instrumentos y los actores. Se trata de determinar los mecanismos a través de los cuales se transmiten los efectos del programa sobre los centros de I+D. Adicionalmente, se integra la perspectiva de las interacciones entre los actores y los instrumentos para observar los modos en que éstas generan efectos en los resultados del programa.

Aunque este es un trabajo descriptivo y analítico (y no deductivo a partir de hipótesis elaboradas sobre teorías fuertes -que no existen en esta literatura que opera más sobre la base de «prueba y error»-), tenemos dos preguntas de investigación para visibilizar nuestros objetivos. La primera, de carácter analítico,

1 En el contexto de nuestro estudio, la noción de «excelencia» se relacionaría grosso modo con la búsqueda, por parte tanto de los actores políticos como de las organizaciones productoras de conocimiento (universidades, agencias, centros de investigación...), de resultados de investigación de muy buena calidad (Jackson y Rushton, 1987). Sabemos que han surgido multiplicidad de concepciones, valores y criterios para definir y juzgar la excelencia (Langfeldt et al., 2020; Thomas et al., 2020) y que, siendo la definición de excelencia fuertemente contextual (Tijssen, 2003), ésta varía en función de las disciplinas y los programas científicos. No obstante, y a pesar de tratarse de un significante controvertido (Hellström, 2011; Hellström, 2018; Hellström et al., 2018), actualmente se observa cierta estandarización del mismo (Deem et al., 2008). Básicamente, se fundamenta en la capacidad de lograr unos resultados «excepcionales» (OECD, 2014). 
GAPP. Nueva Época - N. 28, marzo 2022 - ISSN: 1989-8991 - DOI: https://doi.org/10.24965/gapp.i28.10955 - [Págs. 48-70]

Políticas de investigación para las universidades: La financiación de centros de investigación en Galicia

Manuel Pereira-Puga / Luis Sanz-Menéndez

se refiere a cuáles son los mecanismos esenciales sobre los que el programa quiere apoyar o apalancar el proceso de cambio; tanto las condiciones suficientes (por ejemplo, la financiación adicional que se otorga) como las condiciones necesarias (por ejemplo, un liderazgo o dirección científica).

TABLA 1. Dimensiones de un Centro de inVestigación y CRiterios SEleccionados

\begin{tabular}{l|l}
\hline \multicolumn{1}{c}{ Dimensión del centro de investigación } & \multicolumn{1}{c}{ Atributos de excelencia } \\
\hline Recursos humanos académicos & $\begin{array}{l}\text { Capacidad para atraer investigadores punteros } \\
\text { Existencia de procesos de reclutamiento altamente competitivos }\end{array}$ \\
\hline Financiación y sostenibilidad & $\begin{array}{l}\text { Viabilidad para operar a largo plazo y diversificación de las fuentes } \\
\text { de financiación }\end{array}$ \\
\hline Estructura organizativa & Alto grado de autonomía organizativa y toma de decisiones \\
\hline Liderazgo científico y dirección estratégica & $\begin{array}{l}\text { Selección basada en el mérito y adecuación } \\
\text { Mecanismos que fortalezcan la autoridad científica del director } \\
\text { del centro }\end{array}$ \\
\hline Coordinación y colaboración científica & $\begin{array}{l}\text { Adquisición y uso eficaz y eficiente de instalaciones } \\
\text { y equipamientos }\end{array}$ \\
\hline
\end{tabular}

Fuente: Elaboración propia.

La segunda pregunta de investigación se refiere a la identificación de algunos frenos a la eficacia de los mecanismos, que resultan de la insuficiente coherencia de la cartera de instrumentos y de los efectos contradictorios que otros instrumentos producen. También se aborda el análisis de los obstáculos que surgen del actual marco institucional y modelo de gobernanza de la universidad. Para responder a estas preguntas se describe previamente el programa, junto con sus objetivos explícitos.

\section{EL CASO DE ESTUDIO}

Tras las primeras políticas de I+D de las CC.AA (Cruz-Castro et al., 2004; Sanz-Menéndez y CruzCastro, 2005), se puede decir que algunos gobiernos regionales (como Cataluña, País Vasco y Madrid) optaron por crear centros de I+D, al margen de las universidades y Organismos Públicos de Investigación (OPI) estatales, aunque con diversos grados de vinculación o colaboración con las universidades (SanzMenéndez y Cruz-Castro, 2010). Hemos denominado a esta modalidad de actuación de los gobiernos "cambiar la universidad desde fuera», porque los efectos de «modernización universitaria» se pueden producir por la imitación o influencia indirecta, dado que los sectores más productivos de las universidades acaban teniendo espacios «propios» para la investigación y, por tanto, más capacidad de definir su proyecto. Sin embargo, estas estrategias se han visto por parte de algunas universidades como una cierta descapitalización de sus recursos.

El caso gallego es singular en el contexto español no solo por la larga tradición de una política de I+D (Fernández et al., 2003; Varela-Vázquez et al., 2021), sino porque la política de I+D y los instrumentos implementados en la última década se dirigen a «cambiar la universidad desde dentro». Se han empleado instrumentos de política de I+D con el objetivo de tratar de solucionar problemas identificados, como la fragmentación y dispersión de las capacidades y recursos en las universidades (Pereira-Puga y Sanz-Menéndez, 2020). Asimismo, se ha buscado mejorar las condiciones de trabajo de los profesores e investigadores más dinámicos, de movilizarlos para apoyar desde dentro la mejora de la universidad pública, manteniendo las inversiones hacia ella, aunque -de forma creciente- con objetivos fijados programáticamente.

El caso gallego es relevante también porque Galicia es uno de los casos, si no el único, en que el gobierno autonómico ha aprobado marcos estables plurianuales de financiación universitaria que (incluso en el contexto de la gran recesión) se han negociado o consultado y, sobre todo, se han seguido implementando. Así, las universidades públicas han tenido una envolvente de financiación y de transferencias directas relativamente estable y predecible.

La tercera característica singular tiene que ver con la propia organización de la política de I+D y de la política universitaria dentro de los gobiernos. En las Administraciones Públicas tradicionalmente han sido políticas y áreas diferenciadas, universidad por un lado e I+D por otro, aunque a veces han coincidido bajo el 
mismo ministro o consejero. En general, el dilema para la política de I+D, en cuanto a sus destinatarios, ha sido sector público versus empresas (Cruz-Castro y Sanz-Menéndez, 2021). En Galicia, la política de I+D se ha desdoblado entre política de I+D solo para las universidades (y articulada junto con la política y la financiación de las universidades) y política de I+D de carácter general, que ha estado en otra consejería. Esta última ha elaborado Planes similares a los Estatales desde 1999 (Fernández et al., 2003; González-López y Guntín-Araújo, 2019); véase, por ejemplo, el Plan Gallego de Investigación, Innovación y Crecimiento (I2C) 2011-2015, gestionado por GAIN (Agencia Gallega de Innovación) o la Ley de fomento de la investigación y de la innovación de Galicia ${ }^{2}$, que actúa como marco institucional en este campo.

Así pues, el mecanismo de cambio de la universidad (la financiación de la I+D) se ha integrado instrumentalmente en la política universitaria. Y, de hecho, como se observará en la sección 4.1, es la parte de la financiación universitaria que ha crecido más de forma continuada en la última década, reforzada por los fondos europeos. En definitiva, analizar el caso gallego es útil para comprender mejor los factores que operan en las estrategias de los gobiernos cuando quieren transformar las universidades, sobre todo porque puede ser considerado un caso «divergente» o «extremo» [divergent] (Seawright et al., 2014) en la distribución de las políticas de las CC.AA. Esto puede permitir someter a test empírico la robustez de las teorías o explicaciones aceptadas y los modelos interpretativos dominantes.

\section{APROXIMACIÓN METODOLÓGICA}

Metodológicamente, la aproximación tomada en este trabajo es esencialmente cualitativa y procesual. Aunque el análisis es un caso de estudio (relativo a la política e instrumentos de política pública de I+D), se han realizado comparaciones controladas con las actuaciones de otras CC.AA, en la medida en que el caso se presenta como un caso «extremo» en la distribución.

Los métodos y técnicas utilizados han sido los propios de los enfoques cualitativos de investigación: análisis documentales y presupuestarios, así como entrevistas y reuniones con los actores políticos y administrativos y del propio sistema universitario. Ello con un enfoque esencialmente "descriptivo» de los instrumentos y formas de intervención gubernamentales en este ámbito, pero a la vez integrando las observaciones en el modelo analítico presentado.

Concretamente, se realizaron doce entrevistas semiestructuradas. Se entrevistó a los directores científicos de los centros (en dos de ellos también se entrevistó al vicedirector) y a un representante del gobierno universitario de cada universidad gallega (dos de ellos eran vicerrectores). Asimismo, se mantuvieron reuniones de grupo con investigadores de los centros, que incluyeron en total a treinta científicos. A su vez se mantuvieron múltiples reuniones con responsables de la política de I+D universitaria en la Xunta de Galicia, en concreto en la Secretaria General de Universidades. El trabajo básico de entrevistas se realizó entre los días 7 y 15 de noviembre de 2017, aunque se continuaron las interacciones con los actores a lo largo de 2018, 2019 y 2020.

Junto con ello, ha sido necesario obtener información sobre el contexto financiero del sistema universitario gallego. Específicamente, se han examinado todas las Leyes de Presupuestos de Galicia desde el año 2007 hasta 2021. Adicionalmente, se han analizado todas las convocatorias y resoluciones autonómicas relativas a instrumentos de financiación competitiva de la I+D universitaria publicadas durante el período de análisis, teniendo especialmente en cuenta aquellas destinadas a centros y unidades de I+D.

\section{LA FINANCIACIÓN DE LA UNIVERSIDAD Y DE SU INVESTIGACIÓN POR LA XUNTA DE GALICIA}

La práctica de financiación universitaria en Galicia sigue las tendencias detectadas a nivel global (SanzMenéndez, 2020b): un peso cada vez mayor de la proporción de financiación asignada competitivamente, frente a una financiación basal (vía transferencias directas de la Xunta de Galicia a las universidades) que, si bien no encaja estrictamente en el modelo de financiación basada en resultados, contiene indicadores de resultados como elementos de asignación y distribución de fondos. En esta sección se describe la financiación del SUG, en la que se sitúa el programa de centros universitarios de investigación, así como el resto de programas de financiación competitiva de la I+D universitaria.

\footnotetext{
2 Ley 5/2013, de 30 de mayo, de fomento de la investigación y de la innovación de Galicia (BOE de 9 de julio de 2013 ).
} 


\subsection{La financiación regular de las universidades gallegas}

Galicia cuenta, desde hace más de tres décadas, con planes plurianuales de financiación del SUG, que se desarrollan anualmente en las leyes de presupuestos de la Xunta de Galicia. Desde el año 1990 han estado en vigor hasta seis planes (los correspondientes a 1990-1996, 1996-1999, 2000-2003, 20052010, 2011-2015 y 2016-2020). Estos siguen una evolución incremental de los fondos y una asignación de recursos a las tres universidades basada en el número de estudiantes y el grado de experimentalidad de las titulaciones (Fernández y Vaquero, 2013: Fernández y Vaquero, 2017). El plan 2011-2015 supuso una cierta ruptura con los anteriores al introducir un nuevo modelo de asignación de fondos con dos grandes bloques, financiación estructural y financiación por resultados, referidos siempre a parámetros del conjunto de cada universidad. El Plan 2016-202033, que ha sido prorrogado para $2021^{4}$, planeaba un gasto de 2.069,26 millones de euros para el período (Xunta de Galicia, 2015). Éste ha seguido grosso modo el patrón del anterior. El bloque de financiación estructural es el más importante y sus fondos van destinados a la docencia. Por su parte, la financiación por resultados incluye cuatro sub-fondos, uno de los cuales, denominado «panel de indicadores», recoge 12 indicadores para medir el rendimiento de las universidades en docencia, investigación, transferencia e internacionalización (Fernández y Vaquero, 2017).

A continuación, se analizan los recursos del sistema a través de las leyes de presupuestos generales de la Comunidad Autónoma. En los últimos años se observa un aumento significativo de los fondos destinados a investigación. Estos pasaron, en el período 2008-2021, del $3 \%$ al $18 \%$ de los fondos totales destinados al SUG (véase el gráfico 1).

\section{GRÁfico 1. FinANCIACIÓn POR PROGRAMA PRESUPUESTARIO (2006-2021): OBJETIVOS (IZQUIERDA, EN \%) Y PRESUPUESTO TOTAL (DERECHA), EN MILLONES DE EUROS}

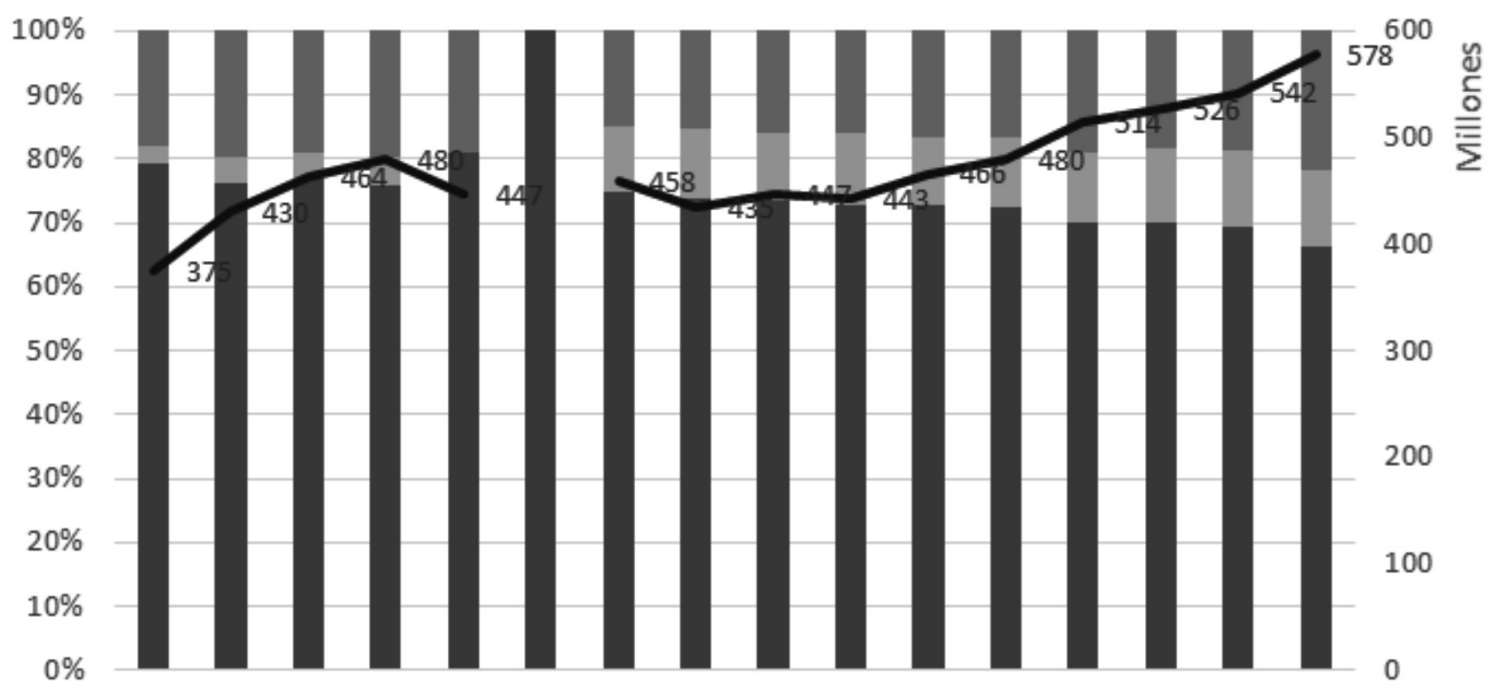

2006200720082009201020112012201320142015201620172018201920202021

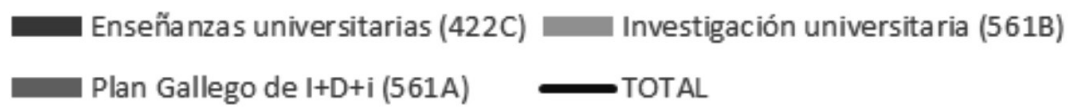

Fuente: Leyes de presupuestos generales de la Comunidad Autónoma de Galicia, varios años.

Nota: Cálculo base sobre agregación de los totales de los programas presupuestarios asignados a la Consejería responsable. Los totales de 2011 corresponden solo a enseñanzas universitarias (422C).

3 Acuerdo del Consejo de la Xunta de Galicia de 29 de octubre de 2015, por el que se aprueba el Plan Gallego de Financiación universitaria 2016-2020 (Resolución de 10 de noviembre de 2015, de la Secretaría General de Universidades, por la que se ordena la publicación del Acuerdo del Consejo de la Xunta de Galicia de 29 de octubre de 2015, por el que se aprueba el Plan gallego de financiación universitaria 2016-2020) (DOG 19 noviembre de 2015).

4 Por la modificación del artículo 115 de la Ley 6/2013, de 13 de junio, del Sistema universitario de Galicia, incluida a través de la Ley $7 / 2019$ de Medidas Fiscales y Administrativas (artículo 36). Este texto legal prevé la prórroga del plan en vigor hasta la aprobación del siguiente (DOG de 27 diciembre 2019). 


\subsection{La financiación competitiva de la I+D universitaria y sus instrumentos}

Los fondos de investigación para las universidades se asignan fundamentalmente a través de programas competitivos destinados a investigadores, grupos de investigación o centros y unidades de investigación. A partir del año 2006, y en un contexto de caída en el esfuerzo de I+D en Galicia (Sande y Vence, 2019, p. 101), la Xunta de Galicia decidió comenzar a financiar competitivamente a grupos de investigación como vía para intentar solucionar problemas diagnosticados en el sistema; entre ellos, la falta de «masa crítica», debida a la fragmentación de las capacidades de investigación (Pereira-Puga y Sanz-Menéndez, 2020). Según los decisores públicos, los investigadores se organizaban en grupos demasiado pequeños y los equipamientos e instalaciones se encontraban dispersos en el sistema, generando ineficiencias. Para revertir esta situación, se implementaron ayudas a grupos de investigación y, a partir de 2008, subvenciones a unidades de investigación, que se sumaron a otras ayudas destinadas a proyectos de investigación y a recursos humanos (pre y postdoctorales).

Así pues, el cambio institucional se produce por acumulación de instrumentos (policy layering) (Thelen 2003; Rayner y Howlett, 2009), donde aparecen nuevas modalidades que se suman a los existentes con anterioridad. En la actualidad, la Xunta convoca regularmente distintas modalidades de ayudas plurianuales para la contratación de recursos humanos (investigadores pre y postdoctorales), realización de proyectos de investigación y, con un peso muy importante, financiación estructural de los grupos de investigación (denominadas ayudas para Grupos de Referencia Competitiva y Grupos con Potencial Crecimiento). La cartera de instrumentos de la Secretaría General de Universidades de la Xunta que se presenta en la tabla 2 incluye la distribución presupuestaria de las ayudas a investigadores y grupos de investigación correspondiente al año 2017, que alcanzó la cifra total de más de 37 millones de euros, incluyendo los compromisos de gasto plurianual.

TAbla 2. Ayudas a investigadores y grupos de investigación de la Secretaría Xeral de Universidades CONVOCADAS EN 2017

\begin{tabular}{|c|c|c|c|c|c|c|}
\hline & \multicolumn{6}{|c|}{ Crédito (en miles de euros) } \\
\hline & 2017 & 2018 & 2019 & 2020 & 2021 & TOTAL \\
\hline Grupos de Referencia Competitiva* & 3.700 & 3.700 & 3.700 & 3.700 & & 14.800 \\
\hline Grupos con Potencial Crecimiento* & 1.200 & 2.100 & 2.100 & & & 5.400 \\
\hline Redes de investigación* & 300 & 600 & 300 & & & 1.200 \\
\hline Proyectos de investigación* & 275 & 425 & 375 & 300 & 200 & 1.575 \\
\hline SUBTOTAL & & & & & & 22.975 \\
\hline $\begin{array}{l}\text { Ayudas para tesis en línea } \\
\text { con los retos de la RIS3*}\end{array}$ & 441,6 & 1.450 & 1.225 & 783,3 & & 3.900 \\
\hline $\begin{array}{l}\text { Ayudas de carácter general de apoyo } \\
\text { a la etapa predoctoral }{ }^{*}\end{array}$ & 313,33 & 1.040 & 860 & 547 & & 2.760 \\
\hline SUBTOTAL & & & & & & 6.660 \\
\hline $\begin{array}{l}\text { Contratos postdoctorales } 2 \text { años } \\
\text { en el extranjero + } 1 \text { año en Galicia* }\end{array}$ & 892,5 & $2.047,5$ & $1.912,5$ & 997,5 & & 5.850 \\
\hline $\begin{array}{l}\text { Contratos postdoctorales } 2 \text { años } \\
\text { en el SUG + ayuda complementaria* }\end{array}$ & 437 & 980 & 643 & & & 2.060 \\
\hline SUBTOTAL & & & & & & 7.910 \\
\hline
\end{tabular}

* No se incluyen los fondos destinados a ayudas para entidades no universitarias (OPIs, fundaciones, etc.).

Fuente: Convocatorias de las ayudas, año 2017. 


\section{LA FINANCIACIÓN AUTONÓMICA DE LOS CENTROS UNIVERSITARIOS DE INVESTIGACIÓN}

\subsection{Antecedentes}

Desde 2008 existe un instrumento para financiar estructuras de investigación denominadas «agrupaciones estratégicas» que se ha convocado en diversas ocasiones: 2008, 2009, 2012, 2015, 2018. Con este instrumento se buscaba financiar la creación de estructuras de investigación «organizadas con un nivel de agregación superior al grupo de investigación que [...] tengan por objetivo desarrollar una actividad colaborativa estable y planificada con objetivos de I+D y transferencia propios» ${ }^{5}$. Esto traería beneficios diversos a la ciencia realizada en las universidades del SUG.

Se pretendía que, al menos, algunas de estas nuevas unidades de I+D (que no se esperaba tuviesen unas estructuras claramente definidas) pudiesen evolucionar organizativa y operativamente hasta convertirse en centros de investigación al uso. Desde 2008, el programa ha sufrido distintas modificaciones en aspectos como: duración (entre dos y tres años), cuantía asignada, requisitos de elegibilidad, tipo de propuesta financiada ${ }^{6}$, entre otros. En la última convocatoria, cada unidad de I+D reconocida como «agrupación estratégica» podía recibir de la Xunta hasta 600.000 euros para su funcionamiento y actividades científicas. Las principales características de este instrumento se recogen en la Tabla 3.

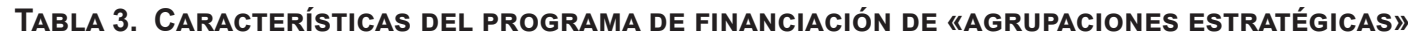

\begin{tabular}{|c|c|c|c|c|c|c|}
\hline $\begin{array}{c}\text { Nombre de la } \\
\text { ayuda }\end{array}$ & $\begin{array}{l}\text { Años en los } \\
\text { que se han } \\
\text { convocado }\end{array}$ & Objetivo & $\begin{array}{c}\text { Duración } \\
\text { (anualidades) }\end{array}$ & $\begin{array}{l}\text { Cuantías de } \\
\text { referencia } \\
\text { en la última } \\
\text { convocatoria (para } \\
\text { todo el período } \\
\text { de duración de la } \\
\text { ayuda euros) }\end{array}$ & $\begin{array}{l}\text { Número de } \\
\text { beneficiarios } \\
\text { en } 2018\end{array}$ & $\begin{array}{c}\text { Total de fondos } \\
\text { adjudicados en } \\
\text { la convocatoria } \\
2018^{*} \\
\text { (puede incluir } \\
\text { anualidades de } \\
\text { años posteriores) }\end{array}$ \\
\hline $\begin{array}{l}\text { Agrupaciones } \\
\text { estratégicas }\end{array}$ & $\begin{array}{l}2008 \\
2009 \\
2012 \\
2015 \\
2018\end{array}$ & $\begin{array}{c}\text { Creación y } \\
\text { consolidación } \\
\text { de unidades } \\
\text { de I+D }\end{array}$ & $2 / 3$ & $\begin{array}{l}\text { Máximo de entre } \\
500.000 € \text { (nuevas } \\
\text { agrupaciones) } \\
\text { y } 600.000 € \\
\text { (agrupaciones } \\
\text { financiadas con } \\
\text { anterioridad) }\end{array}$ & 7 & 3.275 \\
\hline
\end{tabular}

* En miles de euros.

Fuente: Elaboración propia a partir de las órdenes por las que se convocan de las ayudas.

Las siete agrupaciones estratégicas reconocidas en 2018 fueron evaluadas en 2020 por un panel independiente, en el marco del proceso de gestión de las ayudas ${ }^{7}$, y su financiación tiene previsto finalizar a partir de 2021. Quizás esto puede entenderse como un mecanismo de la administración autonómica para reordenar la cartera de instrumentos de actuación en este campo.

\subsection{El programa de centros de investigación singulares del SUG}

Llegado el año 2016, la Xunta de Galicia implementó el «Programa de Centros de Investigación Singulares y Agrupaciones Estratégicas Consolidadas» ${ }^{8}$. Éste estaba destinado a financiar centros de I+D y su objetivo declarado era consolidar unidades de I+D cuyo germen estuviese en una de las agrupaciones

5 DOG núm. 163. Lunes, 25 de agosto de 2008.

6 La convocatoria de 2018 establece dos modalidades distintas: una «Modalidad A» para agrupaciones estratégicas que ya habían sido financiadas en la convocatoria anterior (2015) y una «Modalidad B» destinada a la financiación de nuevas propuestas (DOG núm. 134. Viernes, 13 de julio de 2018).

7 Instrucción 5/2020, de 15 de octubre, de la secretaría general de universidades para iniciar el procedimiento de evaluación del rendimiento de las agrupaciones estratégicas financiadas a través de las ayudas para la creación, reconocimiento y estructuración de agrupaciones estratégicas en el sistema universitario de Galicia, convocadas en el 2018 (https://www.edu.xunta.gal/portal/node/25477).

8 DOG núm. 164. Miércoles, 31 de agosto de 2016. 
estratégicas financiadas con anterioridad a 20149; se quería promover su evolución a formas y modelos organizativos más parecidos a los centros de I+D, con una gobernanza diferenciada de la universidad y una dirección científica más robusta.

El nuevo formato del instrumento, aun teniendo su base en el diagnóstico (fragmentación), recibió la influencia de experiencias de la Administración General del Estado (AGE) y otras CC.AA. El programa "Severo Ochoa y María de Maeztu», servía de modelo sobre el mecanismo de financiación competitiva. Y las actuaciones de otras CC.AA como Cataluña (Centros de Recerca de Catalunya [CERCA]), el País Vasco (Centros de Investigación Cooperativa [CIC]) o Madrid (Instituto Madrileño de Estudios Avanzados [IMDEA]) (González, 2021), pueden entenderse como modelos de centros de I+D hacia los que evolucionar. Sobre la base del aprendizaje de la gestión de los antecedentes, el programa de centros se convirtió en un mecanismo de apoyo al fortalecimiento de la I+D dentro de las universidades; pero con vocación de transformación de las mismas por medio de la investigación y transferencia, en un contexto de reducción del alumnado. En 2016, la Xunta de Galicia asignó casi 14 millones de euros a los siete centros que fueron acreditados para un período de 4 años. Estas ayudas fueron «cofinanciadas por el Fondo Europeo de Desarrollo Regional (FEDER), en el marco del programa operativo FEDER Galicia 2014-2020» y el porcentaje de cofinanciación alcanzó el $80 \%$.

La cuantía máxima de las ayudas podía llegar a los 2,8 millones de euros para cuatro años. La cantidad dada a cada centro venía determinada por la puntuación asignada por el panel evaluador. Nótese que, aunque el programa habla de centros singulares y agrupaciones estratégicas consolidadas, no hay diferencias organizativas ni funcionales entre ambas categorías. Simplemente, los centros mejor evaluados recibirían la acreditación de centro singular, mientras que los que no alcanzasen un determinado umbral serian acreditados como agrupaciones estratégicas consolidadas. Las principales características del programa se resumen en la Tabla 4.

TABLA 4. CARACTERísticas del PROGRAMA DE «CENTROS DE INVESTIGACIÓN SINGULARES Y AGRUPACIONES ESTRATÉGICAS CONSOLIDADAS». FINANCIACIÓN 2016-2019

\begin{tabular}{|c|c|c|c|c|c|}
\hline $\begin{array}{c}\text { Nombre de la } \\
\text { ayuda }\end{array}$ & $\begin{array}{c}\text { Primera } \\
\text { convocatoria }\end{array}$ & Objetivo & $\begin{array}{c}\text { Duración } \\
\text { (anualidades) }\end{array}$ & $\begin{array}{l}\text { Número de } \\
\text { beneficiarios }\end{array}$ & $\begin{array}{l}\text { Total de Fondos } \\
\text { adjudicados, en } \\
\text { millones de euros } \\
\text { (incluye anualidades } \\
\text { de años posteriores) }\end{array}$ \\
\hline $\begin{array}{l}\text { Centros de } \\
\text { investigación } \\
\text { singulares y } \\
\text { agrupaciones } \\
\text { estratégicas } \\
\text { consolidadas }\end{array}$ & 2016 & $\begin{array}{c}\text { Creación y } \\
\text { consolidación } \\
\text { de centros de } \\
\text { investigación } \\
\text { y unidades de } \\
\text { I+D }\end{array}$ & 4 & 7 & $13,274 €$ \\
\hline
\end{tabular}

Fuente: Elaboración propia a partir de la resolución de las ayudas.

Además, se estableció una Comisión asesora del programa (compuesta por representantes de la administración autonómica y científicos destacados ajenos al SUG) que colaboraría con la Consejería en el seguimiento y evaluación de los centros acreditados. Específicamente, y según la orden por la que se convocan las ayudas, la Comisión se encargaría de analizar y evaluar las actuaciones de los centros en distintas dimensiones de interés (incluyéndose la formación, atracción de talento, captación de fondos, calidad de la investigación y otras). A mediados de 2019, un panel independiente llevó a cabo la evaluación de resultados de los tres primeros años, con diversas calificaciones para los centros. En el contexto del proceso se amplió su financiación para el período $2020-2022{ }^{10} \mathrm{y}$, adicionalmente, se incorporó un nuevo centro, el Instituto Ga-

9 La ya mencionada orden por la que se convocan las ayudas exige: «Que la propuesta presentada esté conformada por una unidad supragrupal que hubiese obtenido financiación como agrupación estratégica en una convocatoria de concurrencia competitiva de la Xunta de Galicia anterior a 1 de enero de 2014».

10 DOG núm. 185. Viernes, 11 de septiembre de 2020. 
llego de Física de Altas Energías (IGFAE), que era el único centro de Galicia reconocido, en la convocatoria de 2016, como Unidad María de Maeztu por el Ministerio de Ciencia e Innovación.

Respecto a la organización de estos centros de I+D universitarios, es relevante señalar que (a diferencia de otros) son unidades de las universidades, sin personalidad jurídica propia, ni sistema contable o presupuestario independiente, ni titularidad de las instalaciones que ocupa. Su creación se lleva a cabo según determinan los estatutos de cada universidad, y habitualmente tienen un órgano de gobierno que preside el rector o delegado del rector. Por último, el director es designado, tras un procedimiento de elección, más que selección, por el propio órgano de gobierno.

\subsection{Los centros de investigación universitarios seleccionados y financiados}

A la convocatoria inicial del programa de centros se presentaron ocho solicitudes. Una de ellas fue denegada: el Centro de Investigaciones Agroambientales y Alimentarias (CIA), de la Universidad de Vigo (UVigo), situado en Ourense. Cinco fueron acreditadas como «centros de investigación singulares de Galicia». De la Universidad de Santiago de Compostela (USC): el Centro de Investigación en Química Biológica y Materiales Moleculares (CIQUS) y el Centro de Investigación en Medicina Molecular y Enfermedades Crónicas (CIMUS). De la Universidad de A Coruña (UDC): el Centro de Investigación en Tecnologías de la Información y las Comunicaciones (CITIC); y de la UVigo: el Centro de Investigaciones Biomédicas (CINBIO) y el Centro de Investigaciones Marinas (CIM, anteriormente ECIMAT). Asimismo, dos unidades obtuvieron la acreditación de «agrupación estratégica consolidada»: el Centro de Investigación en Tecnologías de Telecomunicación (AtlanTTic) (UVigo) y el Centro de Investigación en Tecnología de la Información (CITIUS) de la USC. Las principales características de los centros en lo relativo a sus áreas científicas y tamaño se muestran en la tabla 5.

TABLA 5. CAMPo CiEnTífico Y ESTRUCtURA de LA PLANTILLA INVESTIGADORA DE LOS CENTROS DE I+D

\begin{tabular}{|c|c|c|c|c|c|c|c|}
\hline Centro & Universidad & $\begin{array}{c}\text { Campo científico } \\
\text { principal }\end{array}$ & Catedrático* & Prof. Titular** & $\begin{array}{l}\text { Prof. } \\
\text { Contratado } \\
\text { Doctor }\end{array}$ & $\begin{array}{l}\text { Ramón y } \\
\text { Cajal }\end{array}$ & TOTAL \\
\hline CITIC & UDC & $\begin{array}{l}\text { Tecnologías de } \\
\text { la Información y } \\
\text { Comunicaciones }\end{array}$ & 17 & 66 & 41 & 0 & 124 \\
\hline AtlanTTic & UVigo & $\begin{array}{l}\text { Tecnologías de } \\
\text { la Información y } \\
\text { Comunicaciones }\end{array}$ & 17 & 44 & 18 & 1 & 80 \\
\hline ECIMAT/CIM & UVigo & Ciencias del Mar & 17 & 40 & 10 & 3 & 70 \\
\hline CINBIO & UVigo & Ciencias de la salud & 16 & 31 & 20 & 1 & 68 \\
\hline CIMUS & USC & Biomedicina y salud & 12 & 14 & 12 & 4 & 42 \\
\hline CIQUS & USC & $\begin{array}{l}\text { Química y } \\
\text { Bioquímica }\end{array}$ & 12 & 16 & 2 & 3 & 33 \\
\hline CITIUS & USC & $\begin{array}{l}\text { Tecnologías de la } \\
\text { información }\end{array}$ & 4 & 22 & 5 & 1 & 32 \\
\hline TOTAL & & & 95 & 219 & 122 & 13 & 449 \\
\hline
\end{tabular}

* La categoría «catedrático» incluye a Catedráticos de Universidad y de Escuela Universitaria.

** La categoría «Prof. Titular» incluye a Titulares de Universidad y de Escuela Universitaria.

Fuente: Elaboración propia a partir de las plantillas del personal de las unidades.

Por su parte, la distribución de los recursos entre los centros acreditados para el período 2016-2019 puede verse en la Tabla 6. 
GAPP. Nueva Época - N. ${ }^{\circ}$ 28, marzo 2022 - ISSN: 1989-8991 - DOI: https://doi.org/10.24965/gapp.i28.10955 - [Págs. 48-70]

Políticas de investigación para las universidades: La financiación de centros de investigación en Galicia Manuel Pereira-Puga / Luis Sanz-Menéndez

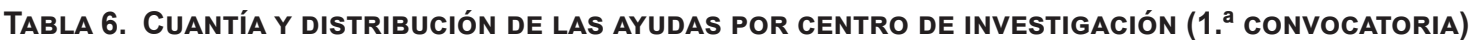
(EN MILES DE EUROS)

\begin{tabular}{|c|c|c|c|c|c|c|c|}
\hline Unidad & Universidad & Categoría & 2016 & 2017 & 2018 & 2019 & TOTAL $€$ \\
\hline CIQUS & USC & $\mathrm{CIS}$ & 400 & 800 & 800 & 800 & 2.800 \\
\hline CIMUS & USC & CIS & 360 & 720 & 720 & 720 & 2.520 \\
\hline CITIC & UDC & $\mathrm{CIS}$ & 255 & 510 & 510 & 510 & 1.785 \\
\hline CINBIO & UVigo & $\mathrm{CIS}$ & 250 & 500 & 500 & 500 & 1.750 \\
\hline ECIMAT/CIM & UVigo & CIS & 250 & 500 & 500 & 500 & 1.750 \\
\hline CITIUS & USC & AEC & 200 & 400 & 400 & 400 & 1.400 \\
\hline AtlanTTic & UVigo & AEC & 181,25 & 362,5 & 362,5 & 362,5 & $1.268,75$ \\
\hline TOTAL & & & $1.896,25$ & $3.792,5$ & $3.792,5$ & $3.792,5$ & $13.273,75$ \\
\hline
\end{tabular}

Fuente: Resolución de la convocatoria.

Como se ha mencionado, la convocatoria de ampliación del programa (que no es el objeto de este análisis) renovó la acreditación a las 7 unidades e integró también al IGFAE. Las ayudas concedidas pueden verse en la tabla 7.

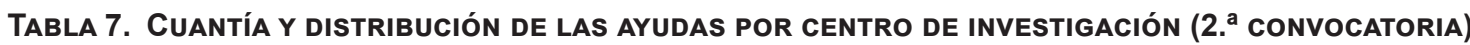
(EN MILES DE EUROS)

\begin{tabular}{l|l|c|c|c|c|c}
\hline \multicolumn{1}{c}{ Centro } & Universidad & 2019 & 2020 & 2021 & 2022 & TOTAL \\
\hline IGFAE & USC & 10 & 1.000 & 1.000 & 1.000 & 3.010 \\
\hline CIQUS & USC & 9,67 & 960 & 960 & 960 & $2.889,67$ \\
\hline CIMUS & USC & 9,33 & 920 & 920 & 920 & $2.769,33$ \\
\hline CITIUS & USC & 7,78 & 733,33 & 733,33 & 733,33 & $2.207,78$ \\
\hline CITIC & UDC & 7,78 & 733,33 & 733,33 & 733,33 & $2.207,78$ \\
\hline CINBIO & UVigo & 7,11 & 653,33 & 653,33 & 653,33 & $1.967,11$ \\
\hline CIM & UVigo & 6,11 & 533,33 & 533,33 & 533,33 & $1.606,11$ \\
\hline AtlanTTic & UVigo & 5,22 & 426,67 & 426,67 & 426,67 & $1.285,22$ \\
\hline
\end{tabular}

Fuente: Resolución de la convocatoria.

\section{ANÁLISIS Y RESULTADOS}

En esta sección se analizan los aspectos más relevantes del diseño del instrumento de financiación de los centros universitarios de I+D, así como los obstáculos que derivados de las restricciones institucionales del modelo de gobernanza y de la coherencia en la cartera de instrumentos frenan el avance de los objetivos. 


\subsection{Los mecanismos del programa}

Desde hace más de quince años las actuaciones de la Secretaría General de Universidades en materia de fomento de la I+D universitaria han estado guiadas por la identificación de un problema estructural: la fragmentación de las actividades de I+D y la falta de masa crítica. Sobre esa lógica se construyeron los programas de financiación de los grupos de investigación como células básicas del sistema que, combinadas en centros de I+D con medios e infraestructuras propias, permitirían consolidar en las universidades una investigación «de calidad» y que, además, «contribuya al desarrollo económico y social de Galicia». Así pues, en el discurso sobre el programa se habla de «agregación» de grupos como parte de un proceso para la construcción y consolidación de centros de I+D, que se ven como el lugar natural de la investigación.

La reducción de la fragmentación y la consolidación de organizaciones especializadas aparece como un dispositivo de mejora de las universidades gallegas y palanca en la competición reputacional de los rankings universitarios. La oportunidad de llevar adelante el ambicioso plan surgió en el contexto de la generosa ayuda europea procedente de los fondos estructurales, que se utilizaron para promover la investigación y la innovación, para la construcción de infraestructuras científicas (edificios, laboratorios, equipamiento, entre otros), así como para la contratación y la formación.

Mientras tanto, la actividad de auto-organización de las universidades proseguía y continuaba con la creación de centros e institutos universitarios, habitualmente surgidos a iniciativa de los propios académicos y dentro de la lógica de las coaliciones de gobierno que se forman en los rectorados. A mediados de la década pasada ya había en las universidades gallegas más «institutos» o «centros» financiables (en 2021, la USC cuenta con 28 unidades de I+D, la UDC con 11 y la UVigo con 6 centros y agrupaciones).

En la fundamentación del programa de centros, la financiación se asociaba al cumplimiento de algunas condiciones adicionales, que debían ser analizadas y evaluadas por paneles independientes. No se trataba solo de centros con «edificios o instalaciones propias delimitadas» o «infraestructuras científicas propias» y (relativamente) «autónomos» o, al menos, diferenciados, del funcionamiento universitario. El énfasis, quizás derivado de los frecuentes contactos y colaboración de los gestores de la Xunta con los responsables de los institutos CERCA en Cataluña, estaba en la definición de un «proyecto científico» (en lenguaje administrativo, un Plan Estratégico) y la identificación y nombramiento de «un director científico» creíble, avalado por la comunidad científica nacional e internacional, y con los mejores resultados en investigación.

Así pues, existe un modelo de centro de I+D subyacente que se caracteriza por un proyecto científico ambicioso, que desborda la simple agregación de los proyectos de los investigadores y académicos que se incorporan, y que está liderado por el mejor científico disponible que debería ser el director del mismo, siempre apoyado por un Comité Científico Asesor externo. Los medios que el programa pone son los recursos financieros adicionales otorgados al centro, en manos del director, que tienen que servir para resolver internamente los problemas de coordinación de las agendas científicas y de acción colectiva.

El programa no solo necesita que el director sea «fuerte», para incentivar y coordinar a los grupos de investigación del centro, sino que necesita que el centro (gracias a los recursos y la visibilidad reputacional) alcance una cierta autonomía y reconocimiento en las estructuras universitarias. Además, con el respaldo político y financiero plurianual de la Xunta y el apoyo exterior de la comunidad científica se espera que se consolide como «espacio protegido» (Whitley, 2014) dentro de la universidad.

\subsection{Los obstáculos al avance de los objetivos del programa}

Sin embargo, esta estrategia de potenciar la «autonomía» y capacidad de decisión de los centros en el contexto de sus universidades, y de fortalecer la coordinación y la dirección de la investigación por los líderes científicos, se enfrenta a constricciones institucionales y obstáculos para la eficiencia del instrumento y el avance de los objetivos.

Los resultados del análisis se organizan en torno a las dos dimensiones propuestas en el enfoque analítico. En primer lugar, se trata cómo el modelo de gobernanza de la universidad y los arreglos institucionales de las universidades de A Coruña, Santiago de Compostela y Vigo para los centros financiados por la Xunta condicionan la capacidad del programa de trasformar las estructuras de investigación. A continuación, se analizan los efectos que tienen sobre el programa el resto de los instrumentos de financiación disponibles y la influencia de los actores del sistema. Todo ello se hace en base a las cinco dimensiones identificadas, sobre las que el programa de centros habría de impactar (recursos humanos, financiación y sostenibilidad, estructura organizativa de los centros, liderazgo científico y colaboración científica), aunque centrándonos 
en el aspecto crítico de cualquier centro de investigación, que es la captación, retención y gestión de los recursos humanos.

\subsubsection{La influencia de la gobernanza y los arreglos institucionales}

Como se ha visto en la descripción del programa, la Xunta promueve la «autonomía» por medio de la financiación directa y competitiva de los centros, pero éstos operan bajo las reglas generales de la universidad, lo que plantea algunas limitaciones importantes. Examinemos pues, a continuación, estas restricciones institucionales al «buen» funcionamiento de los centros.

El modelo de gobernanza de las universidades tiene efectos en las políticas de recursos humanos de los centros, una de las piezas clave de cualquier organización científica. Los directores científicos señalan una capacidad insuficiente de los centros en la captación de talento externo. Esta falta de competitividad percibida se atribuye a déficits que surgen de la gobernanza. Por un lado, a la financiación del Sistema Universitario Español (SUE) y, por el otro, a los arreglos institucionales relativos a la relación de los centros con su universidad matriz. Respecto al primer factor, se subrayan los salarios, inferiores a los de otros países del entorno, y la disponibilidad limitada de recursos materiales y humanos. Esto supondría una barrera al desarrollo de una política propia de captación y retención de talento:

«(Ha habido) investigadores a los que le ofertaban (para su grupo) un pre-doc, un post-doc

y un técnico de por vida. $Y$ un salario que no era... Y nosotros eso no podemos hacerlo». [E2]

Por su parte, las prácticas institucionalizadas en las tres universidades y, aparentemente, la correlación de fuerzas entre departamentos universitarios y centros de investigación impiden que puedan crearse y convocarse plazas permanentes propias o asignadas a estos últimos, que carecen de Relaciones de Puestos de Trabajo (RPT) propias. En el modelo de gobernanza común en la universidad pública española, la convocatoria de plazas fijas de Personal Docente e Investigador (PDI) (Profesor Contratado Doctor, Profesor Titular de Universidad y Catedrático de Universidad) obedece en última instancia a negociaciones internas entre el gobierno de la Universidad y los departamentos (véase, por ejemplo, Cruz-Castro y Sanz-Menéndez, 2015).

La consecución de plazas permanentes de personal científico se produce en el marco del departamento y no en el del centro de investigación. Esto hace que los centros no puedan garantizar a potenciales candidatos una plaza permanente a medio plazo, aunque sus resultados sean excelentes, lo que genera incertidumbre tanto en los investigadores ya adscritos a los centros como en los equipos y la dirección, que han de planificar el funcionamiento del centro:

«Las universidades no se han comprometido a estabilizar a los investigadores de los centros

[...]. Esto destroza los equipos». [E4]

Los centros de investigación no tienen puestos propios (en ninguno de los tipos de personal: científico, técnico y administrativo/servicios), más allá de los profesionales contratados temporalmente con cargo a proyectos o con los fondos del propio programa; y no resulta evidente que, a corto plazo, pueda producirse un cambio en el sentido de dotar a los centros de personal propio.

El modelo de gobernanza actual hace emerger conflictos organizativos derivados de la pluralidad de misiones (docencia, investigación y transferencia) de las universidades. Por un lado, la estabilización en general estaría mucho más ligada a las necesidades docentes que a la investigación; y, por el otro, puede ocurrir que las áreas de mayor fortaleza y potencial investigador no sean necesariamente las que tienen más necesidades docentes. De este modo, investigadores vinculados a centros pueden ver mermadas sus posibilidades de consolidación:

«La actividad investigadora todavía en la Universidad española no forma parte de los elementos que impulsan la contratación (permanente) de personal; son las necesidades docentes las que determinan la contratación de personal, no las necesidades investigadoras». [E1]

Este modelo de gobernanza genera una dependencia de recursos ajenos al sistema; en este caso de los programas competitivos nacionales (singularmente el Ramón y Cajal) e internacionales (European Research Council, ERC) y de la consiguiente negociación ad hoc con un departamento:

«No podemos decir, oye, nos interesa traer a este (menciona un nombre), lo vamos a fichar.

Eso no lo podemos hacer, ¿entiendes? Identificamos una necesidad del centro en (da el nom- 
bre del área); yo quiero fichar a un (nombre del tipo de experto) y no puedo. Tengo que buscarlo a través de un Programa Ramón y Cajal y después que la Universidad me permita, a través de un departamento, la incorporación, etc.». [E5]

Bien es verdad que los cambios introducidos a partir de la Ley de Presupuestos Generales del Estado para 2018, sobre la Oferta de Empleo Público (OEP) (Ley 8/2018, art. 19.3.I), fijaban la reserva de un porcentaje mínimo del $15 \%$ del total de las plazas para investigadores con acreditación certificada I3, habitualmente en manos investigadores Ramón y Cajal o similar, que han pasado la evaluación positiva, lo que puede contribuir a cambiar las perspectivas (Sanz-Menéndez, 2020a).

El modelo de financiación universitaria también interviene en la capacidad de los centros para garantizar su financiación y sostenibilidad y para asegurar su capacidad para operar a largo plazo. Dado un programa de financiación competitiva de organizaciones científicas que concede ayudas por un período de tiempo finito, como suele ser el caso de este tipo de iniciativas (véase, por ejemplo, OECD, 2014), la viabilidad de los proyectos no puede garantizarse en ausencia de una cierta garantía de la financiación estructural. Esta puede darse por la universidad matriz, por otro agente del sistema (como el gobierno regional) o provenir de un compromiso en la obtención de fondos competitivos por parte de los investigadores, lo que evidencia la centralidad de la política de recursos humanos.

Los centros analizados no podrían sobrevivir sin los fondos recibidos a través del programa ya que una parte significativa de éstos se destina a gastos que podrían considerarse estructurales, como los salarios de técnicos de investigación y la financiación de programas de atracción de talento pre y postdoctoral. Ello incluso cuando las universidades-matriz también contribuyen de forma clave, aunque sean aportaciones «en especies» con personal científico (en su mayoría profesores universitarios), así como la dotación y mantenimiento de instalaciones y equipamientos, financiando los gastos corrientes y de funcionamiento y facilitando el uso de los servicios generales de la institución.

Adicionalmente, hay que señalar otra limitación de gestión; los centros carecen de sistemas de contabilidad propios e independientes de las universidades y, en general, están obligados a trabajar con los diversos servicios generales y centralizados de las universidades. Todos estos aspectos generan un dilema para el decisor público. Por un lado, si deja de financiar los centros éstos podrían desaparecer. Por otro lado, dado un contexto de limitación de los recursos públicos, si continúa financiándolos no estará en disposición de promover la puesta en marcha de otras iniciativas.

En el caso de estudio se observa cómo en un contexto financiero de limitación de fondos para I+D, y en ausencia de una garantía de financiación estructural estable por parte de algún organismo (sea un gobierno, fundación, universidad), los actores con responsabilidades en los centros (los directores) rechazan, en general, avanzar hacia modelos organizativos caracterizados por niveles elevados de autonomía e independencia con respecto a la universidad matriz. A pesar de que la autonomía organizativa se ha señalado como uno de los atributos necesarios para la excelencia de este tipo de organizaciones, y siendo conocidas las demandas hacia una mayor autonomía organizativa en los centros de investigación y en las universidades (Cruz-Castro y Sanz-Menéndez, 2018), este posicionamiento por parte de los directores de los centros es muy mayoritario.

Se argumenta que asegurar la inyección de fondos anuales suficientes sería inviable en un ecosistema de I+D como el gallego; $y$, asimismo, se perciben dificultades potenciales para configurar una plantilla investigadora competitiva, al margen de las universidades:

\section{«Estamos sujetos a la Universidad para lo bueno y para lo malo». [E3]}

Se da así una paradoja. Por un lado, el discurso mayoritario gira en torno a que, dada la relación actual con la Universidad, los centros no pueden desarrollarse plenamente; pero, por el otro, no son mayoritarias las demandas de un grado de autonomía más elevado. Preguntados sobre ésta, los participantes perciben que los centros acreditados por la Xunta de Galicia permiten modelos de gestión más flexibles y procesos de toma de decisión más ejecutivos que las estructuras departamentales de las universidades. Además, los directores afirman que los centros facilitan una gestión menos «burocrática» y más «ágil» que la de los departamentos; aunque con limitaciones derivadas de su dependencia de los servicios generales de la universidad:

«Nosotros funcionamos de forma bastante autónoma, dentro de lo que son los mecanismos asamblearios (de los departamentos), donde cada decisión que tomas tiene que pasar siete filtros de comisiones, hablar con los sindicatos, etc. Nosotros en ese aspecto somos bastante autónomos, tomamos las decisiones de funcionamiento, dentro de las limitaciones que tenemos». [E5] 
Siendo esto así, la creación de nuevas estructuras de investigación dentro de las universidades podría estar siendo útil, en cierta medida, para solucionar problemas de gestión y toma de decisiones tradicionalmente detectados en la universidad española. Sin embargo, las restricciones generales del marco institucional reducen las posibilidades de éxito de los objetivos del programa.

\subsubsection{Interacciones entre actores $y$ entre instrumentos}

Ya se ha mencionado que la condición de avance del modelo de centro es la consolidación de una posición institucional en el centro (es decir, la posición del director científico) que contribuya a resolver la acción colectiva y la coordinación. En lo relativo al liderazgo científico, hay que señalar que no hay elementos que garanticen la selección de un líder científico en todas las futuras designaciones de directores. Los directores de los centros, de acuerdo con los estatutos vigentes en las tres universidades, son designados por los Rectores de las mismas, que incluso presiden sus órganos superiores. En algunos hay procesos formales de votación, y no siempre los lideres científicos tienen interés en asumir esta tarea o sus colegas están dispuestos a permitirlo.

En la consideración del liderazgo científico, es necesario tener presente que la autoridad, además de estructural, es relacional, por lo que el control de fondos de investigación se torna en una herramienta clave del liderazgo en el marco de las organizaciones científicas (Cruz-Castro y Sanz-Menéndez, 2018). Cabría esperar que los casi 14 millones de euros inyectados en los centros de investigación con el programa fortaleciesen la autoridad relativa de los directores de los centros, al dotarles de la capacidad de gestión de una proporción significativa de fondos de investigación disponibles. Sin embargo, no resulta evidente que esto sea así. Si concebimos la capacidad de decisión sobre el uso de los fondos obtenidos a través del programa de centros como una herramienta de fortalecimiento del liderazgo de las direcciones científicas, sería esperable que los directores gozasen de una situación ventajosa. Sin embargo, como ya se ha visto, la Xunta de Galicia ha asignado también fondos competitivos en el SUG a través de otros instrumentos financieros. Esto ha hecho que algunos líderes de grupos adscritos a los centros puedan gestionar un volumen de fondos similar al de los propios directores científicos de los centros.

Otra vía de fortalecimiento del liderazgo científico de los centros, no ligada a los recursos económicos, sería el nombramiento de directores reconocidos en la comunidad académica por su reputación y excelencia científica. La autoridad viene determinada por la posición en la estructura jerárquica, pero requiere también el reconocimiento por parte de los otros del derecho legítimo para ejercer esa autoridad. Por eso, la reputación del director, entendida como admiración y respeto de sus pares, sería funcional para guiar y coordinar el centro. Ahora bien, una limitación a esta buena práctica deriva del modelo de gobernanza de la universidad española.

Como se ha dicho, el caso de estos centros permite, pero no asegura, el nombramiento como director de un líder científico. El marco normativo de las universidades del SUG dificulta que el nombramiento del director científico (como también el de directores de departamentos, o del propio rector) sea el resultado de un proceso competitivo abierto a candidatos externos a la universidad, como las buenas prácticas internacionales aconsejan. Así, se hace una selección entre los disponibles, puesto que el conjunto de candidatos potenciales se restringe a la propia universidad, más que entre los mejores posibles.

Más allá de la dirección científica, y respecto a la interacción de actores en un mismo centro, la mayoría de los directores reporta mejoras de la eficacia y la eficiencia en el uso de espacios, así como en la adquisición y empleo de materiales y equipamientos en el marco de los centros de investigación, coherentemente con lo detectado en investigaciones previas (OECD, 2014). Esto sería especialmente relevante en las unidades dedicadas a la ciencia experimental, porque se depende de laboratorios y equipos costosos, los cuales, además, requieren mantenimiento y reparaciones periódicamente. Finalmente, los resultados arrojan cierta evidencia sobre las dinámicas de coordinación y colaboración científica en el marco de la financiación competitiva de centros de investigación. En los centros que poseen un edificio propio (cinco de siete) se describen nuevas prácticas organizativas de adquisición de equipamientos y suministros como, por ejemplo: compras comunes de suministros (con el objetivo adquirir un mayor volumen, rebajando el coste) y de equipamientos (que no podrían alcanzarse en el marco de un único grupo); «laboratorios en abierto» (que cualquier investigador adscrito al centro puede usar); «laboratorios compartidos entre varios grupos», entre otros. Por tanto, y siempre según los entrevistados, la organización de la actividad científica en centros de investigación supondría una ventaja muy destacada con respecto a la situación organizativa anterior en todo lo relativo a las instalaciones y equipamientos. 


\section{CONCLUSIONES}

Los programas que asignan financiación pública para la creación y/o funcionamiento de centros de investigación son instrumentos empleados por gobiernos y agencias de financiación. Ahora bien, más allá de la deseabilidad que una parte de la comunidad académica atribuye a estas políticas y de los efectos positivos esperados, sabemos poco sobre los factores que facilitan y los que dificultan que estos programas sean útiles de cara al éxito de este tipo de organizaciones científicas y al avance de los objetivos fijados.

El análisis del programa de centros de investigación singulares y agrupaciones estratégicas consolidadas de Galicia permite obtener evidencia empírica novedosa sobre cómo el diseño de los instrumentos públicos de financiación de centros, junto con el modelo de gobernanza universitaria y los arreglos institucionales, condicionan los atributos y el funcionamiento de los centros de investigación financiados. Identificando las dimensiones clave de un centro de investigación (recursos humanos, financiación y sostenibilidad, estructura organizativa, liderazgo, e instalaciones y equipamientos), hemos llegado a algunas conclusiones.

En el trabajo hemos analizado la fundamentación y los mecanismos por los cuales se espera que el programa desarrolle sus efectos sistémicos y, adicionalmente, las barreras institucionales y de políticas a los cambios institucionales esperados. En las consecuencias para la acción pública se puede concluir que los programas de financiación de centros de investigación universitarios han de crear una estructura de incentivos que favorezca cambios organizativos funcionales, tanto entre el personal adscrito a los propios centros como en el gobierno universitario. Resulta esencial que los centros puedan desarrollar su propia política de recursos humanos. Esto puede lograrse a través de un arreglo institucional con la universidad matriz (abriendo plazas adscritas a los centros; es decir, que no estén vinculadas a departamentos), a través de la implementación de un instrumento de política pública para la atracción y consolidación de talento, o de otras vías (véanse iniciativas como ICREA e Ikerbasque de los gobiernos catalán y vasco).

Los programas de centros han de fomentar el liderazgo científico incentivando las mejores prácticas internacionales (nombramiento de un líder científico, tras expresión de intereses, en un proceso abierto a académicos externos a la Universidad) e implantando mecanismos fortalecedores del liderazgo (por ejemplo, capacidad de decisión del director sobre áreas de investigación, contrataciones o uso de los fondos). Respecto de la financiación y la capacidad de operar a largo plazo, antes de asignar financiación a un centro sería necesario estudiar cómo va a mantenerse en funcionamiento la organización una vez finalizada la ayuda. Nuevamente, puede llevarse a cabo un arreglo con la universidad matriz, prever un marco de acuerdo entre una administración (regional o estatal) y el centro o buscar algún tipo de colaboración público-privada, entre otras opciones.

La promoción de la autonomía organizativa y la dotación de infraestructuras y equipamientos adecuados para realizar investigación de frontera y/o transferencia de conocimiento al tejido productivo requieren, a su vez, grandes cantidades de recursos humanos (personal técnico, administrativo y de gestión, etc.) y económicos. Nuevamente, esto solo puede conseguirse a través de acuerdos entre el organismo financiador del programa (en este caso el gobierno autonómico) y la universidad matriz. Junto con ello, centro y universidad han de ser capaces de servirse de otros instrumentos disponibles al mismo y otros niveles de gobierno (estatal y europeo), como pueden ser los de compras de equipamiento del estado, o los fondos del FEDER. Desde la perspectiva del decisor público, los instrumentos diseñados e implementados han de ser coherentes (evitando efectos indeseados y potenciales contradicciones derivadas de la acumulación de instrumentos) y no deberían diseñarse individual, sino conjuntamente, en términos de carteras de instrumentos; buscando sinergias y efectos multiplicadores.

Una vez extraídas estas conclusiones, debe referirse que este estudio adolece de ciertas limitaciones. Sería deseable contar con información precisa y fiable sobre los costes totales de cada uno de los centros (personal, gastos corrientes, inversión en infraestructuras y equipamientos, entre otros) y saber qué organismo financia qué y a través de qué fórmulas. Igualmente, habría que obtener información rigurosa sobre el total de fondos competitivos y contractuales obtenidos por todos los investigadores adscritos a los centros. Ello permitiría estudiar en mayor detalle la viabilidad de los centros y, por otro lado, la distribución de autoridad y poder derivada del control de los recursos económicos de la organización.

Para un mayor conocimiento sobre el funcionamiento de los centros de investigación financiados sería necesario profundizar en el análisis sobre la distribución de poder en estas estructuras (entre directores científicos, líderes de grupo e investigadores); derivada de los tres factores identificados en la literatura: posición formal en la jerarquía, gestión de recursos económicos y liderazgo científico (Cruz-Castro y Sanz-Menéndez, 2018). Por otro lado, es conveniente entender mejor las relaciones entre los centros y las universidades que los acogen. Asumiendo que las universidades están compuestas por diferentes actores con prioridades 
y preferencias diversas en términos de asignación de recursos, respuestas a las demandas del entorno, transformaciones organizativas, actividades y áreas de investigación prioritarias, entre otras cuestiones, investigaciones futuras han de determinar la influencia de los diversos actores universitarios en el desarrollo y funcionamiento de centros en proyecto.

Asimismo, no puede obviarse que la puesta en marcha de centros de investigación busca mejorar la creación y/o trasferencia de conocimiento al entorno. Por tanto, entre otras actividades de seguimiento, han de acometerse estudios rigurosos sobre sus resultados de investigación; por ejemplo, su producción científica y tecnológica, las citas recibidas, la evolución de sus redes de colaboración y su participación en proyectos de I+D internacionales, entre otros aspectos. Y, a ser posible, comparando con poblaciones de centros similares, no sometidos al estímulo o bajo otros modelos de gobernanza. Puesto que el trabajo de campo de nuestro estudio se realizó durante el primer período de financiación de los centros (2016-2019) todavía no ha sido posible medir el impacto de la pertenencia al centro en los profesores adscritos a los mismos (en publicación en revistas de alto factor de impacto, artículos altamente citados, colaboración internacional, y otros indicadores). Además, sería necesario estudiar el desempeño diferencial entre centros en función de sus atributos y características organizativas y financieras (controlando por área de investigación).

Finalmente, sería importante integrar en este tipo de análisis la dimensión de los mecanismos y procesos de evaluación del funcionamiento, actividades y rendimiento de los centros en sus distintas formas; singularmente, los comités científicos externos de los propios centros, los mecanismos de evaluación institucional de las universidades y los procesos evaluativos de la Xunta, como financiadora del programa.

\section{AGRADECIMIENTOS}

Este artículo es un resultado de investigación de los trabajos derivados de dos contratos de apoyo tecnológico entre la Secretaría General de Universidades de la Xunta de Galicia y el Instituto de Políticas y Bienes Públicos (IPP) del CSIC. Luis Sanz Menéndez es, desde 2018, miembro de la Comisión Asesora del programa de Centros de Investigación Singulares y Agrupaciones Estratégicas Consolidadas del Sistema Universitario de Galicia (DOG, 11 agosto de 2016). Manuel Pereira Puga es beneficiario de la Ayuda PTA2020-019237-I financiada por MCIN/AEI/ 10.13039/501100011033. Los autores manifiestan no tener conflicto de interés y señalan que sus análisis son independientes del financiador del estudio. Se agradecen las sugerencias recibidas desde el consejo editorial de GAPP y de los dos revisores, así como de Faustino Infante, de la SXU de la Xunta de Galicia. Los errores que pudieran persistir son de responsabilidad de los autores.

\section{ANEXO}

TABLA 8. LISTADO DE ENTREVISTAS

\begin{tabular}{ll}
\hline \multicolumn{1}{c}{ Perfil } & Código entrevista \\
\hline Dirección científica de centro de investigación & E1 \\
\hline Dirección científica de centro de investigación & E2 \\
\hline Dirección científica de centro de investigación & E3 \\
\hline Dirección científica de centro de investigación & E4 \\
\hline Dirección científica de centro de investigación & E5 \\
\hline Dirección científica de centro de investigación & E6 \\
\hline Dirección científica de centro de investigación & E7 \\
\hline Representante de la universidad & E8 \\
\hline Dirección científica de centro de investigación & E9 \\
\hline Representante de la universidad & E10 \\
\hline Dirección científica de centro de investigación & E11 \\
\hline Representante de la universidad & E12 \\
\hline
\end{tabular}




\section{REFERENCIAS BIBLIOGRÁFICAS}

Aagaard, K., Hansen, H. F. y Rasmussen, J. G. (2016). Mergers between governmental research institutes and Universities in the Danish HE sector. European Journal of Higher Education, 6(1), 41-55. https://doi.org/10.1080/2 1568235.2015.1099459

Arenilla Sáez, M. (coord.) (2012). La reforma de la universidad española: Un análisis desde su gobernanza. Netbiblo.

Balderston, F. E. (1995). Managing Today's University: Strategies for Viability, Change, and Excellence. Jossey-Bass Higher and Adult Education Series. ERIC.

Baldridge, J. V. (ed.). (1971). Academic Governance - Research on Institutional Politics and Decision Making. McCutchan Pub. Corp.

Benitez-Amado, A. (2019). El gobierno de las Universidades en España. Análisis comparativo, transformaciones recientes y adaptación al entorno [tesis doctoral]. UAM. Departamento de Ciencia Política y Relaciones Internacionales; CSIC. Instituto de Políticas y Bienes Públicos (IPP). https://repositorio.uam.es/ handle/10486/689278

Bennett, C. J. y Howlett, M. (1992). The lessons of learning: Reconciling theories of policy learning and policy change. Policy Sciences, 25(3), 275-294. https://doi.org/10.1007/BF00138786

Bleiklie, I., Enders, J., Lepori, B. y Musselin, C. (2011). New Public Management, network Governance and the University as a Changing Professional Organization. En T. Christensen y P. Laegreid (eds.), The Ashgate Research Companion to New Public Management (1. ${ }^{a}$ ed., pp. 161-176). Routledge. https://hal-sciencespo. archives-ouvertes.fr/hal-00972968

Borrás, S. (2012). Three tensions in the governance of science and technology. En D. Levi-Faur (ed.), The Oxford handbook of governance (1. ${ }^{a}$ ed., pp. 429-440). Oxford University Press. https://doi.org/10.1093/ oxfordhb/9780199560530.013.0030

Bozeman, B. y Boardman, P. C. (2003). Managing the New Multipurpose, Multidiscipline University Research Center: Institutional Innovation in the Academic Community. IBM Center for The Business of Government. https://www. businessofgovernment.org/report/managing-new-multipurpose-multidiscipline-university-research-centerinstitutional-innovation

Braun, D. (1993). Who Governs Intermediary Agencies? Principal-Agent Relations in Research Policy-Making. Journal of Public Policy, 13(2), 135-162. https://doi.org/10.1017/S0143814X00000994

Capano, G. (2018). Policy design spaces in reforming governance in higher education: The dynamics in Italy and the Netherlands. Higher Education, 75(4), 675-694. https://doi.org/10.1007/s10734-017-0158-5

Capano, G., Pritoni, A. y Vicentini, G. (2020). Do policy instruments matter? Governments' choice of policy mix and higher education performance in Western Europe. Journal of Public Policy, 40(3), 375-401. https://doi.org/10.1017/ S0143814X19000047

Cocos, M. y Lepori, B. (2020). What we know about research policy mix. Science and Public Policy, 47(2), 235-245. https://doi.org/10.1093/scipol/scz061

Cohen, M. D., March, J. G. y Olsen, J. P. (1972). A Garbage Can Model of Organizational Choice. Administrative Science Quarterly, 17(1), 1-25. https://doi.org/10.2307/2392088

Cremonini, L., Horlings, E. y Hessels, L. K. (2018). Different recipes for the same dish: Comparing policies for scientific excellence across different countries. Science and Public Policy, 45(2), 232-245. https://doi.org/10.1093/scipol/ $\operatorname{scx} 062$

Cruz-Castro, L. y Sanz-Menéndez, L. (2005). Bringing science and technology human resources back in: The Spanish Ramón y Cajal programme. Science and Public Policy, 32(1), 39-53. https://doi. org/10.3152/147154305781779687

Cruz-Castro, L. y Sanz-Menéndez, L. (2007). Research evaluation in transition. Individual versus organisational assessment in Spain. En R. Whitley y J. Gläser (eds.), The Changing Governance of the Sciences: The Advent of Research Evaluation Systems (pp. 205-223). Springer Netherlands. https://doi.org/10.1007/978-1-40206746-4_10

Cruz-Castro, L. y Sanz-Menéndez, L. (2015). Policy change and differentiated integration: Implementing Spanish higher education reforms. Journal of Contemporary European Research, 11(1), 103-123. https://www.jcer.net/index.php/ jcer/article/view/628

Cruz-Castro, L. y Sanz-Menéndez, L. (2018). Autonomy and Authority in Public Research Organisations: Structure and Funding Factors. Minerva, 56(2), 135-160. https://doi.org/10.1007/s11024-018-9349-1.

Cruz-Castro, L. y Sanz Menéndez, L. (2021). La innovación en la política española. Papeles de Economía Española, 169, 16-35. https://www.funcas.es/articulos/la-innovacion-en-la-politica-espanola/

Cruz-Castro, L., Martínez, C., Peñasco, C. y Sanz-Menéndez, L. (2020). The classification of public research organizations: Taxonomical explorations. Research Evaluation, 29(4), 377-391. https://doi.org/10.1093/reseval/ rvaa013

Cruz-Castro, L., Sanz Menéndez, L. y Romero, M. (2004). Convergencia y divergencia en las políticas de ciencia y tecnología de los gobiernos regionales. Revista Española de Ciencia Política, 11, 33-72. https://recyt.fecyt.es/ index.php/recp/article/view/37356 
De Boer, H. F., Enders, J. y Leisyte, L. (2007). Public Sector Reform in Dutch Higher Education: The Organizational Transformation of the University. Public Administration, 85(1), 27-46. https://doi.org/10.1111/j.14679299.2007.00632.x

Deem, R., Mok, K. H. y Lucas, L. (2008). Transforming higher education in whose image? Exploring the concept of the "world-class" university in Europe and Asia. Higher Education Policy, 21(1), 83-97. https://doi.org/10.1057/ palgrave.hep. 8300179

Fernández López, S. y Vaquero García, A. (2013). The Model Galician University Funding 2011-2015: Provident Fund And Balance. Revista Galega de Economía, 22(1), 73-96. https://doi.org/10.15304/rge.22.1.1270

Fernández López, S. y Vaquero García, A. (2017). Luces y sombras del nuevo plan autonómico de financiación de las universidades gallegas (2016-2020). Revista Galega de Economía, 26(1), 89-104. https://doi.org/10.15304/ rge.26.1.4323

Fernández Mellizo-Soto, M., Sanz Menéndez, L. y Cruz Castro, L. (2003). Diseño institucional y preferencias políticas: O cómo equilibrar los intereses académicos en la política deficiencia, tecnología e innovación gallega. Inguruak. Revista Vasca de Sociologia y Ciencia Política, 35, 33-68.

García-Aracil, A. (2013). Planificación estratégica en la universidad española: Discursos y efectos. Presupuesto y Gasto Público, 72, 67-85. https://www.ief.es/docs/destacados/publicaciones/revistas/pgp/72_04.pdf

González Hermoso, A. (2021). Los nuevos centros públicos no universitarios de investigación: EI Programa IMDEA. Papeles de Economía Española, 169. https://www.funcas.es/articulos/los-nuevos-centros-publicos-nouniversitarios-de-investigacion-el-programa-imdea/

González-López, M. y Guntín-Araújo, X. (2019). Evolución da política galega de innovación: De cero á especialización intelixente. Revista Galega de Economía, 28(2), 23-38. https://doi.org/10.15304/rge.28.2.6154

Hedström, P. (1998). Rational imitation. En P. Hedström y R. Swedberg (eds.), Social Mechanisms: An Analytical Approach to Social Theory (pp. 306-328). Cambridge University Press. https://doi.org/10.1017/ CBO9780511663901.012

Hellström, T. (2011). Homing in on excellence: Dimensions of appraisal in Center of Excellence program evaluations. Evaluation, 17(2), 117-131. https://doi.org/10.1177/1356389011400891

Hellström, T. (2018). Centres of excellence and capacity building: From strategy to impact. Science and Public Policy, 45(4), 543-552. https://doi.org/10.1093/scipol/scx082

Hellström, T., Brattström, E. y Jabrane, L. (2018). Governing interdisciplinary cooperation in Centers of Excellence. Studies in Higher Education, 43(10), 1763-1777. https://doi.org/10.1080/03075079.2018.1520476

Hernández Armenteros, J. y García, J. A. P. (2015). La financiación universitaria como instrumento dinamizador de cambio en la universidad pública española. CIAN-Revista de Historia de Las Universidades, 18(1), 79-96. https:// e-revistas.uc3m.es/index.php/CIAN/article/view/2670

Hicks, D. (2012). Performance-based university research funding systems. Research Policy, 41(2), 251-261. https://doi. org/10.1016/j.respol.2011.09.007

Hood, C. (1983). The Tools of Government. MacMillan Press.

Howlett, M. (2009). Governance modes, policy regimes and operational plans: A multi-level nested model of policy instrument choice and policy design. Policy Sciences, 42(1), 73-89. https://doi.org/10.1007/s11077-009-9079-1

Jackson, D. N. y Rushton, J. (1987). Scientific excellence: Origins and assessment. Sage Publications, Inc.

Jonkers, K. y Zacharewicz, T. (2016). Research performance based funding systems: A comparative assessment. Publications Office of the European Union. https://data.europa.eu/doi/10.2760/70120

Kickert, W. (1995). Steering at a Distance: A New Paradigm of Public Governance in Dutch Higher Education. Governance, 8(1), 135-157. https://doi.org/10.1111/j.1468-0491.1995.tb00202.x

Kingdon, J. W. (1996). Agendas, Alternatives, and Public Policies (2. ${ }^{a}$ ed.). Pearson.

Langfeldt, L., Nedeva, M., Sörlin, S. y Thomas, D. A. (2020). Co-existing notions of research quality: A framework to study context-specific understandings of good research. Minerva, 58(1), 115-137. https://doi.org/10.1007/s11024-019-09385-2

Larrán-Jorge, M. y García-Correas, Á. (2015). ¿Influyen los modelos de financiación autonómicos en la eficiencia de las universidades públicas españolas? Revista de Contabilidad, 18(2), 162-173. https://doi.org/10.1016/j. rcsar.2014.06.001

Lepori, B. (2011). Coordination modes in public funding systems. Research Policy, 40(3), 355-367. https://doi. org/10.1016/j.respol.2010.10.016

Lindblom, C. E. (1959). The Science of "Muddling Through". Public Administration Review, 19(2), 79-88. https://doi. org/10.2307/973677

Marginson, S. (1997). Steering from a distance: Power relations in Australian higher education. Higher Education, 34(1), 63-80. https://doi.org/10.1023/A:1003082922199

Martínez Martínez, R. (2012). Reflexions a l'entorn del sistema de govern de les universitats davant del repte de modernització de la governança universitària. Revista Catalana de Dret Públic, 44. http://revistes.eapc.gencat.cat/ index.php/rcdp/article/view/2240

May, P. J., Jones, B. D., Beem, B. E., Neff-Sharum, E. A. y Poague, M. K. (2005). Policy Coherence and ComponentDriven Policymaking: Arctic Policy in Canada and the United States. Policy Studies Journal, 33(1), 37-63. https:// doi.org/10.1111/j.1541-0072.2005.00091.x 
May, P. J., Sapotichne, J. y Workman, S. (2006). Policy Coherence and Policy Domains. Policy Studies Journal, 34(3), 381-403. https://doi.org/10.1111/j.1541-0072.2006.00178.x

OECD (2008). OECD Reviews of Tertiary Education Tertiary Education for the Knowledge Society. OECD Publishing. https://doi.org/10.1787/19978936

OECD (2014). Promoting Research Excellence: New Approaches to Funding. OECD Publishing. https://doi. org/10.1787/9789264207462-en

Olsen, J. P. (2007). The Institutional Dynamics of the European University. En P. Maassen y J. P. Olsen (eds.), University Dynamics and European Integration (pp. 25-54). Springer Netherlands. https://doi.org/10.1007/978-14020-5971-1_2

Pereira-Puga, M. y Sanz-Menéndez, L. (2020). Cambio y continuidad en las políticas de ciencia: Diseño de los instrumentos de financiación de la investigación universitaria en Galicia (2005-2018). Revista Española de Ciencia Política, 54, 151-171. https://doi.org/10.21308/recp.54.06

Pérez-Esparrells, C., De la Torre, E. M. y Gómez-Sancho, J. M. (2018). La relevancia de la financiación pública en las universidades españolas. Presupuesto y Gasto Público, 90, 169-190. https://www.ief.es/docs/destacados/ publicaciones/revistas/pgp/90_10.pdf

Peters, B. G. (2011). Steering, rowing, drifting, or sinking? Changing patterns of governance. Urban Research \& Practice, 4(1), 5-12. https://doi.org/10.1080/17535069.2011.550493

Rayner, J. y Howlett, M. (2009). Introduction: Understanding integrated policy strategies and their evolution. Policy and Society, 28(2), 99-109. https://doi.org/10.1016/j.polsoc.2009.05.001

Rip, A. (1994). The republic of science in the 1990s. Higher Education, 28(1), 3-23. https://doi.org/10.1007/BF01383569

Rose, R. (2005). Learning From Comparative Public Policy. A Practical Guide. Routledge.

Sahlin, K. y Eriksson-Zetterquist, U. (2016). Collegiality in modern universities - the composition of governance ideals and practices. Nordic Journal of Studies in Educational Policy, 2016(2-3), artículo 33640. https://doi.org/10.3402/ nstep.v2.33640

Sande Veiga, D. y Vence, X. (2019). Impact Evaluation of the Technology Fund Programme 2007-2013 in Galicia: Results, aid concentration and leakage of resources. Revista Galega de Economía, 28(3), 92-114. https://doi. org/10.15304/rge.28.3.5926

Sanz-Menéndez, L. (1997). Estado, ciencia y tecnología en España (1939-1997). Alianza editorial.

Sanz-Menéndez, L. (2020a). La atracción del talento y la política de recursos humanos de las universidades. En E. Alcón Soler, F. Michavila Pitarch y M. Ripollés (eds.), Más talento para la universidad española: Retenerlo, atraerlo, recuperarlo (pp. 159-184). Tecnos.

Sanz-Menéndez, L. (2020b). La financiación de la investigación universitaria y sus resultados desde una perspectiva comparada. Anuario de La Facultad de Derecho de La Universidad Autónoma de Madrid, 24, 109-135. http:// afduam.es/wp-content/uploads/2021/06/luis-sanz-menendez.pdf

Sanz-Menéndez, L. y Cruz-Castro, L. (2005). Explaining the science and technology policies of regional governments. Regional Studies, 39(7), 939-954. https://doi.org/10.1080/00343400500289945

Sanz-Menéndez, L. y Cruz-Castro, L. (eds.) (2010). La investigación y sus actores. Institutos, Centros de I+D y sus desafios (vol. 12/2010). Fundacion CYD. http://2017.fundacioncyd.org/images/documentosCyd/CYD12.pdf

Seawright, J., Gerring, J., Seawright, J. y Gerring, J. (2014). Case Selection Techniques in Case Study Research: A Menu of Qualitative and Quantitative Options. En M. Tight (ed.), Case Studies (pp. II213-II213). SAGE Publications Ltd. https://doi.org/10.4135/9781473915480.n31

Simon, H. A. (1979). Rational Decision Making in Business Organizations. The American Economic Review, 69(4), 493513. https://www.jstor.org/stable/1808698

Strehl, F., Reisinger, S. y Kalatschan, M. (2007). Funding Systems and their Effects on Higher Education Systems (OECD Education Working Paper, 6). OECD Publishing. https://doi.org/10.1787/220244801417

Thelen, K. (2003). How institutions evolve. Insights for comparative historical analysis. En J. Mahoney y D. Rueschemeyer (eds.), Comparative Historical Analysis in the Social Sciences (pp. 208-240). Cambridge University Press; Cambridge Core. https://doi.org/10.1017/CBO9780511803963

Thomas, D. A., Nedeva, M., Tirado, M. M. y Jacob, M. (2020). Changing research on research evaluation: A critical literature review to revisit the agenda. Research Evaluation, 29(3), 275-288. https://doi.org/10.1093/reseval/ rvaa008

Tijssen, R. J. W. (2003). Scoreboards of research excellence. Research Evaluation, 12(2), 91-103. https://doi. org/10.3152/147154403781776690

Van Steen, J. (2012). Modes of Public Funding of Research and Development: Towards Internationally Comparable Indicators (OECD Science, Technology and Industry Working Paper, 2012/04). OECD Publishing. https://doi. org/10.1787/5k98ssns1gzs-en

Varela-Vázquez, P., Carmona-Badía, X., González-López, M. y Guntín-Araújo, X. (2021). Aprendizaje institucional y formación de políticas científico-tecnológicas y de innovación en Galicia: Un enfoque histórico, 1981-2020. Investigaciones de Historia Económica. https://doi.org/10.33231/j.ihe.2021.02.001

Whitley, R. (2007). Changing governance of the public sciences. En The changing governance of the sciences (pp. 3-27). Springer. 
Whitley, R. (2011). Changing Governance and Authority Relations in the Public Sciences. Minerva, 49(4), 359-385. https://doi.org/10.1007/s11024-011-9182-2

Whitley, R. (2014). How do Institutional Changes Affect Scientific Innovations? The Effects of Shifts in Authority Relationships, Protected Space, and Flexibility. En Organizational Transformation and Scientific Change: The Impact of Institutional Restructuring on Universities and Intellectual Innovation (vol. 42, pp. 367-406). Emerald Group Publishing Limited. http://www.emeraldinsight.com/doi/full/10.1108/S0733-558X20140000042012

Xunta de Galicia (2015). Plan Galego de Financiamento Universitario (2016-2020). http://www.edu.xunta.gal/portal/es/ node/17660

Zacharewicz, T., Lepori, B., Reale, E. y Jonkers, K. (2019). Performance-based research funding in EU Member States - A comparative assessment. Science and Public Policy, 46(1), 105-115. https://doi.org/10.1093/scipol/scy041 